\title{
Sentido de vida: una herramienta en la formación de estudiantes universitarios en el sureste de México
}

\author{
The Meaning of Life: A Tool in the Training of University Students in \\ Southeastern Mexico
}

\section{Sentido de vida: uma ferramenta na formação de estudantes universitários no sudeste do México}

\author{
Gladis Ivette Chan Chi \\ Universidad Autónoma de Yucatán, México \\ ivette.chan@correo.uady.mx \\ https://orcid.org/0000-0001-7885-8136
}

\section{Resumen}

El presente estudio tuvo como objetivo diagnosticar el sentido de vida de estudiantes de nuevo ingreso a un campus de ciencias sociales, económico-administrativas y humanidades de una universidad pública en el sureste de México, a fin de desarrollar estrategias que promuevan la atención del sentido de vida: una herramienta útil en la formación de los alumnos, puesto que favorece el desarrollo de las potencialidades, el establecimiento de metas, la satisfacción en las actividades académicas, profesionales y personales, al mismo tiempo que apoya la adaptación y trayectoria escolar de los educandos. Este estudio se desarrolló desde un enfoque metodológico cuantitativo, de tipo descriptivo y diseño no experimental. Se utilizó un muestreo no probabilístico por conveniencia de sujetos voluntarios, por lo que la muestra estuvo conformada de 551 educandos, a quienes se les administró el Purpose in Life Test (PIL) en su versión en español. Los resultados mostraron que $51.5 \%$ (284) presentaba incertidumbre y vacío existencial y solo $48.5 \%$ (267) sentido de vida. Por otro lado, no se encontraron diferencias significativas por programa educativo, por sexo y por edad, lo que indicaba que dichas variables resultaron no ser predictoras de la presencia de sentido de vida en los estudiantes. A partir de los resultados fue posible diseñar 


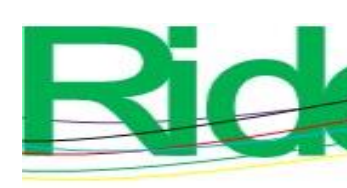

Revista Iberoamericana para la
Investigación y el Desarrollo Educativo
ISSN $2007-7467$

una intervención educativa como estrategia enfocada en la promoción de la búsqueda y fortalecimiento del sentido de vida de los universitarios, con el objetivo de favorecer su adaptación al contexto universitario, contribuir en su formación y apoyarlos en el establecimiento de metas académicas y personales.

Palabras clave: educación superior, estudiantes, formación, logoterapia, sentido de vida.

\section{Abstract}

The present study aimed to diagnose the meaning of life of new students to a campus of social, economic-administrative and humanities sciences of a public university in southeastern Mexico, in order to develop strategies that promote attention to the sense of life: a useful tool in the training of students, since it favors the development of potentialities, the establishment of goals, satisfaction in academic, professional and personal activities, at the same time that it supports the adaptation and school trajectory of students. A nonprobabilistic convenience sampling of volunteer subjects was used, so the sample consisted of 551 students, who were administered the Purpose in Life Test (PIL) in its Spanish version. The results showed that $51.5 \%$ (284) presented uncertainty and existential emptiness and only $48.5 \%$ (267) had a sense of life. On the other hand, no significant differences were found by educational program, by sex and by age, which indicated that these variables were not predictive of the presence of a sense of life in the students. Based on the results, it was possible to design an educational intervention as a strategy focused on promoting the search for and strengthening the meaning of life of university students, with the aim of favoring their adaptation to the university context, contributing to their training and supporting them in the establishment of academic and personal goals.

Keywords: higher education, students, training education, logotherapy, meaning of life.

\section{Resumo}

O presente estudo teve como objetivo diagnosticar o sentido de vida de novos alunos para um campus de ciências sociais, econômico-administrativas e humanas de uma universidade pública no sudeste do México, a fim de desenvolver estratégias que promovam a atenção ao sentido da vida: uma ferramenta útil na formação de alunos, uma vez que favorece o desenvolvimento de potencialidades, o estabelecimento de metas, a satisfação nas atividades acadêmicas, profissionais e pessoais, ao mesmo tempo que apóia a adaptação e a trajetória 


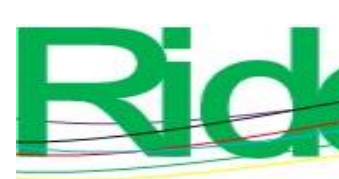

\section{Revista Iberoamericana para la Investigación y el Desarrollo Educativo ISSN $2007-7467$}

escolar dos alunos. Este estudo foi desenvolvido a partir de uma abordagem metodológica quantitativa, do tipo descritivo e com delineamento não experimental. Foi utilizada uma amostra de conveniência não probabilística de voluntários, de forma que a amostra foi composta por 551 alunos, aos quais foi aplicado o Teste de Propósito na Vida (PIL) em sua versão em espanhol. Os resultados mostraram que 51,5\% (284) apresentavam incerteza e vazio existencial e apenas 48,5\% (267) tinham sentido de vida. Por outro lado, não foram encontradas diferenças significativas por programa educacional, por sexo e por idade, o que indica que essas variáveis não são preditivas da presença de sentido de vida nos alunos. A partir dos resultados, foi possível delinear uma intervenção educativa como estratégia voltada para a promoção da busca e fortalecimento do sentido de vida dos universitários, com o objetivo de favorecer sua adaptação ao contexto universitário, contribuir para sua formação e apoiá-los. no estabelecimento, objetivos acadêmicos e pessoais.

Palavras-chave: ensino superior, estudantes, formação, fonoaudiologia, sentido da vida. Fecha Recepción: Noviembre 2020 Fecha Aceptación: Mayo 2021

\section{Introducción}

La escuela debe centrarse en educar con sentido. Según Martínez (2007), la educación debe promover la libertad, la responsabilidad, los valores y la búsqueda del sentido de la vida. El sentido de vida, de acuerdo con Gómez del Campo, Medina y Aguilar (2011), además de proporcionarle un significado a la existencia, le añade satisfacción a las actividades que los seres humanos realizan. Visto de esta manera, se trata del eje central del éxito personal. Martínez, Llantá y Bayarre (2018) afirman que el sentido de vida es aquello que proporciona un significado a la vida y, por consiguiente, favorece el contacto con un soporte interno a la existencia. Por su parte, Frankl (2004) sostiene que el sentido de vida representa una guía para el desarrollo pleno de los seres humanos. Y para Wojtyla (2005), este aparece cuando las personas son capaces de vislumbrar su existencia, resolverla y orientarla.

Esta concepción de sentido de vida deriva de un sistema denominado logoterapia, que significa 'salud a través del sentido'. Para la logoterapia, el sentido de vida es un aspecto clave para vislumbrar de forma positiva la vida. Toda persona tiene la capacidad de encontrarlo, sobreponerse a las diferentes circunstancias que enfrenta y convencerse de que vale la pena vivir (Frankl, citado en Fabry, 2009). Por lo tanto, la logoterapia es una educación para la vida. Evidentemente dicho enfoque prioriza explorar las capacidad 


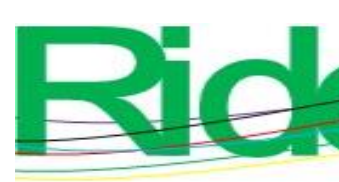

\section{Revista Iberoamericana para la Investigación y el Desarrollo Educativo ISSN $2007-7467$}

humanas y existenciales. Además, ofrece una serie de instrumentos educativos que contribuyen a que los estudiantes se den cuenta de que pueden hacerse cargo de su propia experiencia, única e irrepetible, considerando las condiciones en las que viven para poder proyectarse, dirigirse y valorar su existencia. Desde el punto de vista de Ascencio de García (14 de septiembre de 2009), es fundamental enseñar a los alumnos a cuidarse a sí mismos para comprender su propia vida y proporcionarle un sentido, y al hacerlo también se logrará ayudar a otras personas a comprenderse a sí mismas. "Para cuidar a los otros hay que saber cuidarse a sí mismo" (Ascencio de García, 14 de septiembre de 2009, párr. 54).

De manera que el sentido de vida resulta ser una herramienta útil que puede ser empleada para reforzar la formación de los estudiantes: promover el desarrollo de sus potencialidades y su motivación para el establecimiento de metas, de acciones y compromisos concretos para el logro de cada una de ellas, incluyendo, por supuesto, las actividades académicas.

En esa misma línea, Cadavid y Díaz (2015) apuntan que el sentido de vida se encuentra relacionado con el logro de metas y objetivos, al mismo tiempo que es asociado con el desempeño académico, por lo que un joven que lo ha encontrado es capaz de elegir su carrera profesional por vocación. Castillo (2018) afirma que los jóvenes que logran encontrar un propósito en su vida, además de establecer metas y compromisos, también logran adjudicarle significado y relevancia a las actividades escolares. Este concepto también se encuentra asociado a la resiliencia en los jóvenes universitarios. De hecho, Smedema y Franco (2018) encontraron una correlación positiva significativa entre los niveles de resiliencia y sentido de vida de 215 estudiantes universitarios. Asimismo, García, Martínez, Sellés y Soucase (2018) afirman que el sentido de vida también se encuentra relacionado con los aspectos cognitivos, emocionales y motivacionales, los cuales están enfocados al bienestar subjetivo. Estos aspectos son la autovaloración positiva, la autoaceptación, la libertad, la responsabilidad, la autodeterminación, así como con una visión positiva de la vida, la satisfacción vital, el compromiso con las metas existenciales, la autotrascendencia y el afrontamiento ante la adversidad.

Es así como aquellas personas que han descubierto su sentido de vida son capaces de darse cuenta de los recursos personales y valores que poseen para poder establecer metas en función de sus potencialidades y, a partir de ello, emprender acciones precisas que les permitan trabajar para alcanzar las metas académicas y personales previamente propuestas. Por lo tanto, es un factor protector $y$, al mismo tiempo, una herramienta que puede ser 

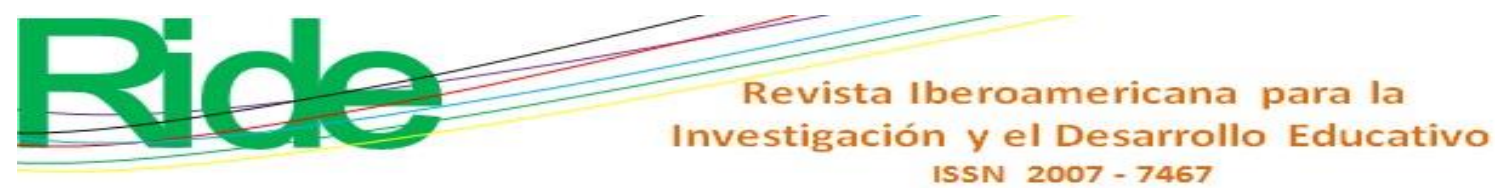

último podría ser debido a la falta de sentido e incluso a la presencia de incertidumbre y vacío existencial. De ahí la importancia de diagnosticar el nivel de sentido de vida que presentan los estudiantes desde su primer ingreso a la universidad, a fin de desarrollar estrategias pertinentes para atender oportunamente este aspecto y contribuir en su formación.

Ahora bien, varias son las investigaciones que adoptan por tema el sentido de vida en los universitarios. A continuación, citaremos algunas de ellas. En primer lugar, Martínez y Castellanos (2013) desarrollaron un estudio cuyo objetivo fue describir la percepción del sentido de vida de universitarios en Bogotá, Colombia. Los resultados mostraron que $36.7 \%$ presentaban bajo sentido de vida; también se encontró que los estudiantes entre 16 y 20 años de edad poseían mayor sentido de vida y que los educandos de programas educativos como medicina y enfermería presentaban altos puntajes de sentido de vida, mientras que los de psicología y comunicación social obtuvieron puntajes significativamente más bajos en comparación con los de otras licenciaturas.

En esta misma línea, Magaña, Zavala, Ibarra, Gómez y Gómez (2004) realizaron un estudio con universitarios de nuevo ingreso a 23 carreras de la Universidad la Salle Bajío. Entre sus objetivos estaba el valorar el sentido de vida de los participantes. Los resultados revelaron que $55.5 \%$ de los alumnos presentaban propósito de vida, $33.3 \%$ indefinición y el $11.2 \%$ vacío existencial. Magaña et al. (2004) refieren la necesidad de atender a la población que presenta indefinición y vacío, puesto que son estudiantes en situación de riesgo y quienes podrían llegar a presentar un desajuste emocional.

Aunado a lo anterior, García, Gallego y Pérez (2009) demostraron que el sentido de vida se asocia de manera positiva a factores como la percepción, libertad, responsabilidad y autodeterminación, al mismo tiempo que les permite a las personas contar con una visión positiva de ellos mismos, de su futuro y también al cumplimiento de sus metas. Finalmente, Damon (2009) desarrolló un estudio donde encontró que los jóvenes que logran definir su sentido de vida encuentran relevancia y significatividad en su experiencia académica, en sus tareas y en las demandas propias del colegio.

Lo anterior permite comprender la relevancia que tiene el constructo sentido de vida como un motivador que impulsa a los educandos para continuar esforzándose en sus estudios y enfrentar, en función de sus recursos personales, las situaciones inherentes a la vida académica, a sus relaciones interpersonales y la etapa de transición en los primeros semestres de la universidad, la cual puede llegar a tornarse confusa debido a los requerimientos del programa educativo, de las nuevas formas de trabajo, la importancia de la toma de decisiones 


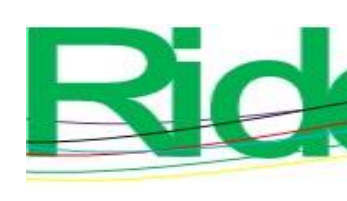

Revista Iberoamericana para la
Investigación y el Desarrollo Educativo
ISSN $2007-7467$

Marco de referencia

\section{El sentido de vida}

La logoterapia concibe el sentido de vida como el significado concreto de la vida de cada individuo en un momento determinado, por un lado, y como la responsabilidad que este tiene para responder en cada situación de su vida, por el otro. De acuerdo con Frankl (1997), es el hombre mismo, guiado por su conciencia, quien tiene que realizar la búsqueda de su sentido de vida para poder encontrar un significado y un propósito. Esta búsqueda favorecerá la madurez, el crecimiento y permitirá la realización del ser humano. Sin embargo, tal y como Andrade (2018) argumenta, el sentido no es estático, sino que cambia constantemente, puesto que, cuando uno se siente realizado, siempre aparece una nueva dirección en la vida de los seres humanos.

A sabiendas de este dinamismo, Fabry (2009) manifiesta que el sentido puede presentarse de dos maneras: el sentido último y el sentido del momento. El sentido último es inalcanzable para cualquier ser humano, puesto que resulta ser como un horizonte hacia el cual se intenta llegar, pero que siempre está un paso por delante de nosotros. El sentido del momento, por el contrario, puede ser alcanzado y es una orientación hacia una existencia plena. Cabello (2000) afirma que la primera acción que debe realizar alguien que busca encontrar el sentido de la vida es hacerse cargo de su propia existencia, por lo que debe aprender a hacerse responsable de las consecuencias de sus actos, puesto que es así cómo se logra obtener la libertad.

Por lo tanto, el sentido de vida surge cuando una persona logra descubrir el significado de su vida y es capaz de establecer propósitos y metas claras, así como acciones y compromisos concretos para trabajar en función de ellos. Además, representa la claridad que tiene el ser humano acerca de la razón por la cual se encuentra en esta vida, de las cosas que puede ofrecer al mundo y también de todo aquello que puede recibir de la vida misma, puesto que quien tiene un sentido es capaz de vislumbrar un motivo claro que lo impulse a seguir adelante y le permita proyectarse como una persona útil.

Es así como se hace evidente que la búsqueda del sentido de vida es responsabilidad única del hombre mismo y solamente encontrándolo podrá otorgarle un significado a su vida, construir su propia existencia, vislumbrar como relevantes cada una de sus actividades escolares, así como darle dirección y orientación a cada una de sus acciones para llegar a las metas propuestas. Como ya se ha mencionado, una persona que ha encontrado el sentido de vida tiende a proyectarse hacia el futuro, construir proyectos, metas e ideas, puesto que se 


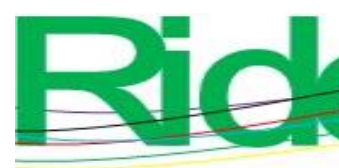

Revista Iberoamericana para la Investigación y el Desarrollo Educativo ISSN $2007-7467$

siente motivado y es capaz de tomar decisiones que impacten en su vida tanto académica como personal.

Educar en lo humano y en la afectividad es imprescindible para afianzar los lazos y vínculos entre las personas. Stramiello (2006) subraya lo fundamental que es orientar a los educandos hacia la responsabilidad, la autonomía, los valores y la búsqueda del sentido de la vida, ya que este último es el motivador por excelencia de cualquier alumno.

\section{Sentido de vida en la formación del estudiante universitario}

Maldonado, Arredondo y de la Garza (2010) afirman que el sentido de vida se encuentra orientado hacia la acción humana, puesto que quien tiene una razón para vivir es capaz de soportar cualquier situación (Nietzsche, citado en Xirau, 1987).

La educación debe promover espacios oportunos que les permitan a los estudiantes ser conscientes de la importancia que tiene el hecho de construir un sentido para su propia vida como un aspecto clave para el éxito tanto personal como profesional (Mallart, Mallart y Valls, 2011). Particularmente, la educación universitaria debe posicionar en el centro la búsqueda del sentido de vida, procurando una formación profesional que involucre tanto el ser como el hacer, donde se cultive, además de la intelectualidad, el crecimiento personal, donde se eduque la libertad para tomar decisiones, asumir la responsabilidad y el desarrollo de actitudes positivas y conductas basadas en los valores, puesto que la combinación de conocimientos teóricos, metodológicos, conceptuales, procedimentales y la visión positiva de la vida, y de que esta vale la pena de ser vivida, favorecerá el crecimiento tanto intelectual como personal.

En suma, la universidad debe formar profesionales capaces de enfrentarse con actitud crítica a los retos y demandas de la sociedad, asumir su libertad, responsabilidad, sus valores éticos para sustentar su propia existencia y, por ende, la misión que la vida le tiene reservada.

Finalmente, contar con un sentido de vida les permite a los estudiantes encontrar relevancia en el desarrollo de sus actividades académicas, favorece el establecimiento de metas, compromisos y potencia la capacidad de aprender a superar obstáculos inherentes a la vida académica, personal y profesional, asumiendo siempre actitudes positivas. De ahí la importancia de encontrar o fortalecer el sentido de vida de los estudiantes desde su primer ingreso al nivel superior, a fin de apoyar su proceso de adaptación y transición en esta nueva etapa, ya que se trata de una herramienta para la formación integral del estudiantado, puesto que permite potenciar el desarrollo de sus capacidades y talentos. Durante su vida tanto 

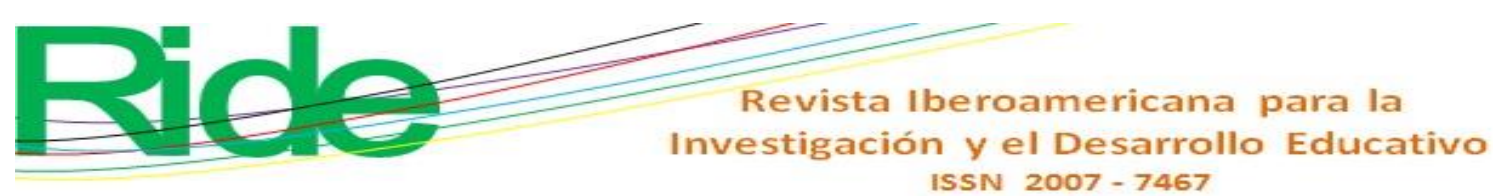

académica como personal, los individuos atravesarán por diferentes obstáculos y depende de ellos exclusivamente y de los recursos personales y académicos que poseen el poder enfrentarlos y continuar avanzando en sus estudios hasta conseguir aquellas metas que se han propuesto.

\section{Metodología}

En el presente estudio se empleó un enfoque cuantitativo, con un estudio descriptivo y un diseño no experimental, debido a que la información referente a la variable fue recolectada en un solo momento. El objetivo de la investigación fue diagnosticar el sentido de vida de los estudiantes de nuevo ingreso al nivel superior de un campus de ciencias sociales, económico-administrativas y humanidades. También, una vez con los datos recolectados, determinar si las variables Programa educativo, Sexo y Edad resultan ser predictoras del sentido de vida en los estudiantes. Y, por último, con base en los resultados del diagnóstico, poder diseñar una intervención educativa como estrategia que contribuya a la atención de este aspecto en la vida de los educandos y favorezca su trayectoria escolar.

\section{Muestra}

La muestra estuvo conformada por 551 estudiantes de nuevo ingreso de un campus de ciencias sociales, económico-administrativas y humanidades de una universidad pública ubicada en el sureste de México. Los alumnos estaban inscritos a las licenciaturas de Historia, Arqueología, Antropología Social, Comunicación Social, Turismo, Literatura Latinoamericana, Psicología, Economía, Comercio Internacional, Derecho, Educación y Enseñanza del Idioma Inglés. En cuanto al género, $58.6 \%$ (323) eran del sexo femenino, mientras que $41.4 \%$ (228) eran del sexo masculino. Las edades de los participantes oscilaban entre los 18 y 38 años.

Se empleó un muestreo no probabilístico por conveniencia de sujetos voluntarios. Los sujetos fueron informados de los objetivos del estudio y las características de la investigación, y decidieron participar libre y voluntariamente, con firma de consentimiento informado de por medio. Se establecieron como criterios de inclusión que los participantes fueran estudiantes con inscripción vigente en los programas de licenciatura ofertados en las dependencias educativas (facultades) ubicadas físicamente en el campus anteriormente mencionado, así como también que decidieran participar libre y voluntariamente. 


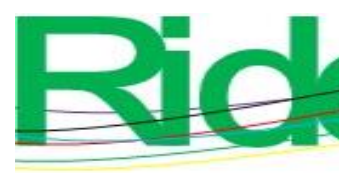

Revista Iberoamericana para la Investigación y el Desarrollo Educativo ISSN $2007-7467$

\section{Instrumento}

En este estudio se utilizó el Purpose in Life Test (PIL), traducido y adaptado al español por Noblejas (2000). El PIL está conformado de tres secciones, pero para efectos de este estudio solamente se empleó la primera sección. Dicha sección está compuesta de 20 reactivos con perfiles de polaridad, construidos a partir de una escala de Gutman del uno al siete, donde la persona debe marcar como respuesta un solo número correspondiente. Cabe aclarar que el número cuatro representa una respuesta neutral, es decir, no indica una toma de decisiones y que los números distribuidos del uno al siete emplean diferentes términos descriptivos, por lo que es fundamental revisar la respuesta que se asigna a cada reactivo para evitar confusiones.

La confiabilidad de este instrumento fue de 0.85 , dato reportado por Crumbaugh (1973), mientras que al ser corregida por Spearman-Brown se obtuvo un nivel de confiabilidad de 0.92. Igualmente, no está de más señalar que este instrumento busca detectar el vacío existencial, la incertidumbre de propósito y el sentido de vida que presentan las personas a través de la suma de las puntuaciones obtenidas en la totalidad del instrumento. De acuerdo con los datos de baremación, la primera parte del instrumento se interpreta de la siguiente forma: una puntuación menor de 92 puntos indica vacío existencial, mientras que una puntuación entre 92 y 112 puntos hace referencia a un dictamen de indefinición con respecto al propósito de vida y, finalmente, una puntuación mayor a 112 puntos significa la presencia de metas y propósitos de vida (Fabry, 2009).

\section{Procedimiento para la recolección y análisis de los datos}

En cuanto al procedimiento para la recolección de los datos, primeramente, se realizó una solicitud a las autoridades de las diferentes dependencias para poder administrar el PIL a los estudiantes de nuevo ingreso, previa explicación de los objetivos del estudio. Y a partir de la autorización de cada dependencia, se proporcionó un calendario de fechas y horarios para administrar el instrumento y también se procedió a firmar el consentimiento informado por parte de los educandos. Posteriormente se elaboró una base de datos y se analizó la información recabada a través del programa Statistical Package for de Social Sciences (SPSS) en su versión número 15, y se emplearon análisis de frecuencias y porcentajes, que permitieron identificar el nivel de sentido de vida de los estudiantes de nuevo ingreso a la universidad. También se realizó el análisis de la varianza (Anova) a través de la prueba 


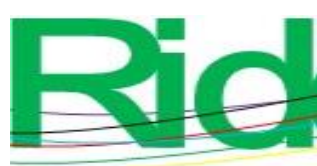

\section{Revista Iberoamericana para la Investigación y el Desarrollo Educativo ISSN $2007-7467$}

paramétrica de un factor. Por último, se utilizó la prueba $t$ de Student para la comparación de medias.

\section{Resultados}

A continuación, se presentan los resultados obtenidos del análisis de los datos que fueron recolectados mediante la administración del PIL a fin de diagnosticar el sentido de vida. Además de la variable Sentido de vida, como ya se mencionó líneas atrás, también se tomaron en cuenta las variables de Programa educativo, Sexo y Edad de los participantes.

Tabla 1. Nivel de sentido de vida de los estudiantes de nuevo ingreso

\begin{tabular}{|l|l|l|}
\hline \multicolumn{1}{|c|}{ Dictamen } & \multicolumn{1}{|c|}{$n$} & \multicolumn{1}{c|}{$\%$} \\
\hline Vacío & 69 & 12.5 \\
\hline Incertidumbre & 215 & 39.0 \\
\hline Propósito de vida & 267 & 48.5 \\
\hline Total & 551 & 100 \\
\hline
\end{tabular}

Fuente: Elaboración propia

En la tabla 1 se puede observar que $51.5 \%$ (284) de los estudiantes presenta un nivel de incertidumbre o vacío existencial, lo que señala la necesidad de apoyar a los estudiantes para fortalecer su sentido de vida, así como para encontrar satisfacción en la realización de sus actividades, apoyar su trayectoria académica y permanencia en la institución. 


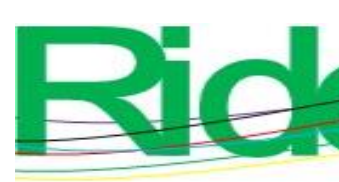

Revista Iberoamericana para la Investigación y el Desarrollo Educativo ISSN $2007-7467$

Tabla 2. Nivel de sentido de vida de los alumnos de nuevo ingreso por programa educativo

\begin{tabular}{|l|l|l|l|l|}
\hline \multirow{2}{*}{ Programa educativo } & \multicolumn{3}{|c|}{ Dictamen } & \\
\cline { 2 - 5 } & \multicolumn{1}{|c|}{ Vacío } & Incertidumbre & Sentido de vida & Total \\
& $\%(\mathrm{n})$ & $\%(\mathrm{n})$ & & \\
\hline Historia & $28.57 \%(6)$ & $47.61 \%(10)$ & $23.80 \%(5)$ & 21 \\
\hline Arqueología & $16.66 \%(3)$ & $44.44 \%(8)$ & $38.88 \%(7)$ & 18 \\
\hline Antropología Social & $15.28 \%(4)$ & $38.46 \%(10)$ & $46.15 \%(12)$ & 26 \\
\hline Psicología & $9.80 \%(10)$ & $40.19 \%(41)$ & $50 \%(51)$ & 102 \\
\hline Derecho & $6.89 \%(6)$ & $43.67 \%(38)$ & $49.42 \%(43)$ & 87 \\
\hline Comunicación Social & $10.71 \%(6)$ & $35.71 \%(20)$ & $53.57 \%(30)$ & 56 \\
\hline Economía & $13.72 \%(7)$ & $31.37 \%(16)$ & $54.90 \%(28)$ & 51 \\
\hline Enseñanza del Idioma & $10.71 \%(3)$ & $32.14 \%(9)$ & $57.14 \%(16)$ & 28 \\
\hline Inglés & & & & \\
\hline Educación & $9.80 \%(5)$ & $31.37 \%(16)$ & $58.82 \%(30)$ & 51 \\
\hline Comercio Internacional & $16.66 \%(9)$ & $38.88 \%(21)$ & $44.44 \%(24)$ & 54 \\
\hline Turismo & $11.42 \%(4)$ & $40 \%(14)$ & $48.57 \%(17)$ & 35 \\
\hline Literatura & $27.27 \%(6)$ & $54.54 \%(12)$ & $18.18 \%(4)$ & 22 \\
\hline Latinoamericana & & & & 551 \\
\hline Total & $12.52 \%(69)$ & $39.01 \%(215)$ & $48.45 \%(267)$ & \\
\hline
\end{tabular}

\section{Fuente: Elaboración propia}

En la tabla 2 se puede observar que existe una mayor proporción de estudiantes con sentido de vida en los programas educativos de Psicología, Comunicación Social, Economía, Enseñanza del Idioma Inglés y Educación, mientras que en las licenciaturas de Historia, Arqueología, Antropología Social, Derecho, Comercio Internacional, Turismo y Literatura Latinoamericana más de $50 \%$ de los educandos presenta incertidumbre y vacío existencial, lo que muestra una clara necesidad de que las dependencias educativas desarrollen estrategias para trabajar en el fortalecimiento de este factor. 


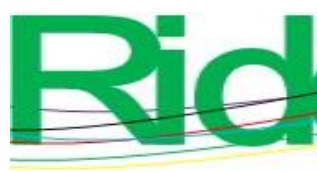

de

Revista Iberoamericana para la

Investigación y el Desarrollo Educativo

ISSN $2007-7467$

Tabla 5. Comparación del puntaje de sentido de vida de los alumnos por sexo

\begin{tabular}{|c|c|c|c|c|c|c|c|c|c|c|}
\hline & \multicolumn{2}{|c|}{$\begin{array}{l}\text { Prueba de Levene } \\
\text { para la igualdad } \\
\text { de varianzas }\end{array}$} & \multicolumn{7}{|c|}{ Prueba $t$ de Student para la igualdad de medias } \\
\hline & & \multirow{2}{*}{$\begin{array}{l}\mathrm{F} \\
\text { Inferior }\end{array}$} & \multirow{2}{*}{\begin{tabular}{|l|} 
Sig. \\
Superior
\end{tabular}} & \multirow{2}{*}{ t } & \multirow{2}{*}{$\begin{array}{l}\text { Gl } \\
\text { Superior }\end{array}$} & \multirow{2}{*}{$\begin{array}{l}\text { Sig. } \\
\text { (bilateral) } \\
\text { Inferior }\end{array}$} & \multirow{2}{*}{$\begin{array}{l}\text { Diferencia } \\
\text { de medias } \\
\text { Superior }\end{array}$} & \multirow{2}{*}{$\begin{array}{l}\text { Error típ. } \\
\text { de la } \\
\text { diferencia } \\
\text { Inferior }\end{array}$} & \multicolumn{2}{|c|}{$\begin{array}{l}95 \% \text { intervalo de } \\
\text { confianza para la } \\
\text { diferencia }\end{array}$} \\
\hline & & & & & & & & & Superior & Inferior \\
\hline 营. & $\begin{array}{l}\text { Se han } \\
\text { asumido } \\
\text { varianzas } \\
\text { iguales }\end{array}$ & 8.794 & .003 & -3.070 & 549 & .002 & -4.069 & 1.325 & -6.672 & -1.466 \\
\hline & $\begin{array}{l}\text { No se } \\
\text { han } \\
\text { asumido } \\
\text { varianzas } \\
\text { iguales }\end{array}$ & & & -2.924 & 397.967 & .004 & -4.069 & 1.392 & -6.805 & -1.333 \\
\hline
\end{tabular}

*P-valor $>0.005$

Fuente: Elaboración propia

Como se puede notar en la tabla 5, se realizó un análisis estadístico de comparación de medias utilizando la prueba $t$ de Student para muestras independientes y se encontró que no existen diferencias significativas entre el nivel de sentido de vida y el sexo de los estudiantes de nuevo ingreso a los programas educativos de licenciatura de un campus de ciencias sociales, económico-administrativas y humanidades de una universidad pública en el sureste de México, lo cual indica que el sexo no se relaciona directamente con el sentido de vida.

De igual forma, se empleó la prueba $t$ de Student para muestras independientes para comparar los puntajes obtenidos en la PIL con la edad de los participantes y los resultados mostraron que no existían una relación significativa entre el sentido de vida y la edad de los alumnos, por lo que no existe relación entre dichas variables $(r=0.078, p>0.05)$. 

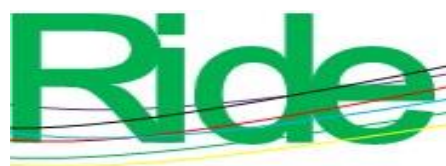

Revista Iberoamericana para la Investigación y el Desarrollo Educativo ISSN $2007-7467$

Con base en los resultados obtenidos, se puede notar que los estudiantes de nivel superior de una universidad pública en el sureste de México tienen una baja percepción de su sentido de vida, e incluso se encontraron dictámenes de estudiantes que presentan vacío existencial, lo cual subraya la necesidad de desarrollar estrategias que permitan atender este aspecto en la vida de los jóvenes, puesto que el sentido de vida es un factor protector que puede servir de apoyo en la adaptación y trayectoria académica de los estudiantes. Así pues, a partir de estos resultados, fue posible diseñar como estrategia una intervención educativa desde la orientación de la logoterapia a fin de trabajar en el fortalecimiento del sentido de vida de los educandos desde su primer ingreso a la universidad

A continuación, se presenta en la tabla 6 de manera sintética el diseño de la propuesta para trabajar en la búsqueda o fortalecimiento del sentido de vida. 
Tabla 6. Descripción sintética de la propuesta

Datos generales de la propuesta

Título: Fortalecimiento del sentido de vida

Dirigido a estudiantes de nuevo ingreso al nivel superior de un campus de ciencias sociales, económicoadministrativas y humanidades de una universidad pública en el sureste de México.

Duración total: 20 horas

Objetivo: Fortalecer el sentido de vida de los estudiantes de nuevo ingreso al nivel superior a través del reconocimiento de sus recursos para el desarrollo de su potencial académico.

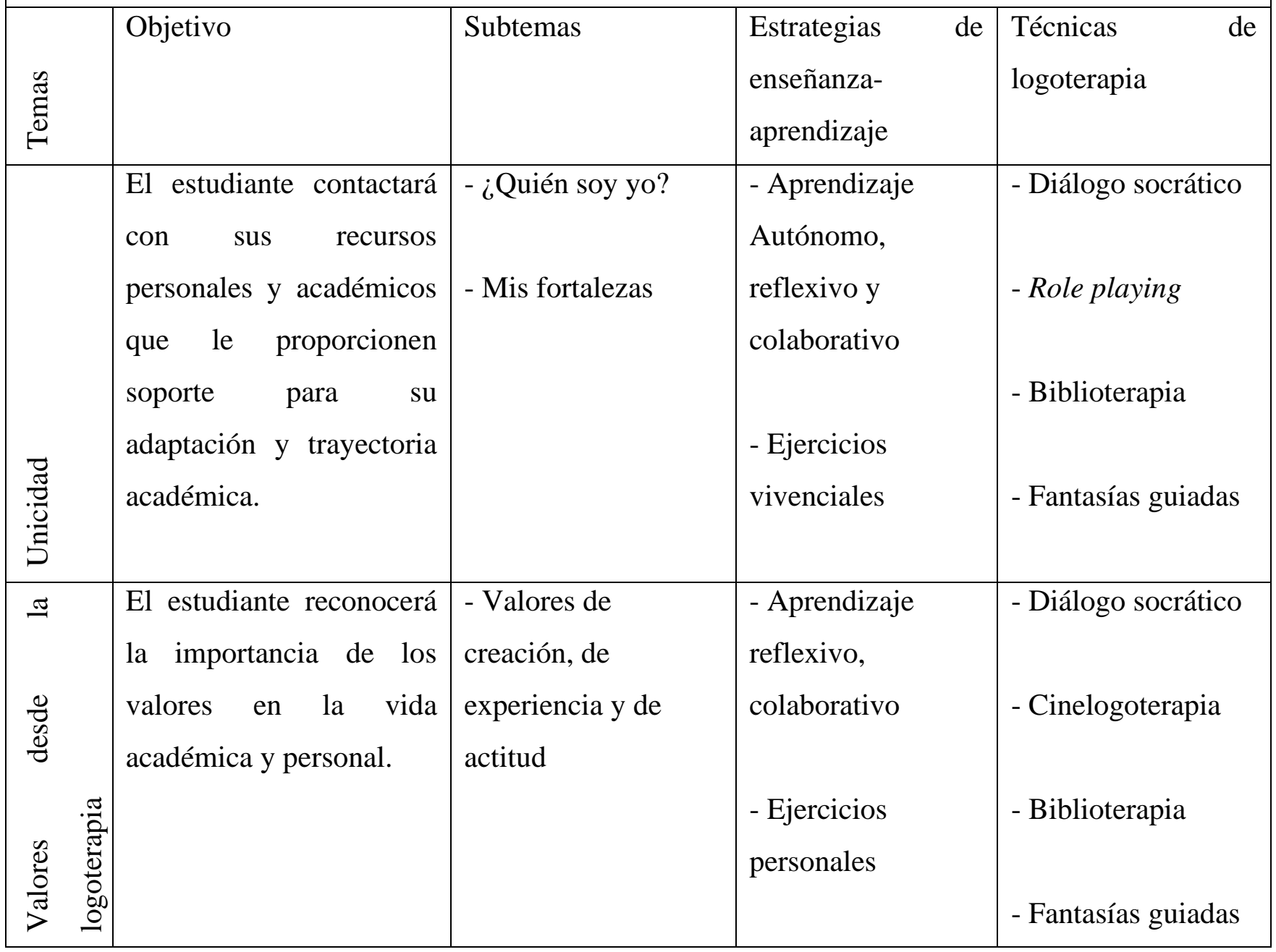




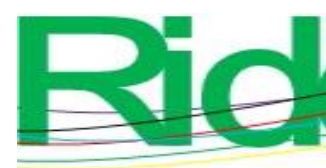

Revista Iberoamericana para la Investigación y el Desarrollo Educativo ISSN 2007 - 7467

\begin{tabular}{|c|c|c|c|c|}
\hline 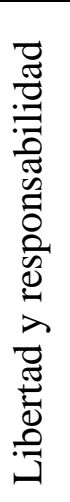 & $\begin{array}{l}\text { El estudiante asumirá la } \\
\text { responsabilidad de la } \\
\text { toma de decisiones que } \\
\text { realice en su vida } \\
\text { académica y personal. }\end{array}$ & $\begin{array}{l}\text { - Condicionamientos } \\
\text { - Voluntad } \\
\text { - Actitudes ante la } \\
\text { vida }\end{array}$ & $\begin{array}{l}\text { - Aprendizaje } \\
\text { reflexivo y } \\
\text { colaborativo } \\
\text { - Ejercicios } \\
\text { vivenciales }\end{array}$ & $\begin{array}{l}\text { - Diálogo socrático } \\
\text { - Cinelogoterapia } \\
\text { - Role playing } \\
\text { - Biblioterapia }\end{array}$ \\
\hline 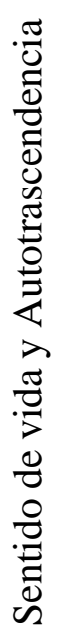 & $\begin{array}{l}\text { El estudiante fortalecerá } \\
\text { su sentido de vida a través } \\
\text { del reconocimiento de sus } \\
\text { potencialidades y metas } \\
\text { para autotrascender a } \\
\text { nivel personal y } \\
\text { académico. }\end{array}$ & $\begin{array}{l}\text { - Establecimiento de } \\
\text { metas con sentido } \\
\text { - La importancia de } \\
\text { la autotrascendencia } \\
\text { en la vida académica } \\
\text { y personal. }\end{array}$ & $\begin{array}{l}\text { - Aprendizaje } \\
\text { reflexivo, } \\
\text { autónomo y } \\
\text { colaborativo } \\
\text { - Ejercicios } \\
\text { vivenciales }\end{array}$ & $\begin{array}{l}\text { - Diálogo socrático } \\
\text { - Cinelogoterapia } \\
\text { - Biblioterapia } \\
\text { - Role playing }\end{array}$ \\
\hline
\end{tabular}

Fuente: Elaboración propia

En la tabla 6 se puede observar de forma general el diseño de la propuesta con enfoque de logoterapia, así como las estrategias de enseñanza-aprendizaje y las técnicas de logoterapia que se han considerado para trabajar en la búsqueda o fortalecimiento del sentido de vida, desde una metodología teórico-vivencial. Resulta evidente que se busca trabajar en el desarrollo de las potencialidades de los educandos, así como en su crecimiento personal, a fin de fortalecer su sentido de vida y, al mismo tiempo, proporcionarles la oportunidad de establecer metas claras que orienten y direccionen su vida, facilitando su adaptación al ámbito universitario, su trayectoria académica, la superación de obstáculos, toma de decisiones, permanencia en la institución y conclusión exitosa de sus estudios.

\section{Discusión}

Los resultados obtenidos con respecto al nivel de sentido de vida de los alumnos indicaron que $51.5 \%$ (284) de los participantes presenta incertidumbre de propósito y vacío existencial, lo cual muestra que la mayoría de los estudiantes aún no logra encontrar un sentido a sus vidas, y que experimentan sentimientos de duda, insatisfacción y frustración. Por consiguiente, no logran construir planes de acción, así como tampoco establecer 


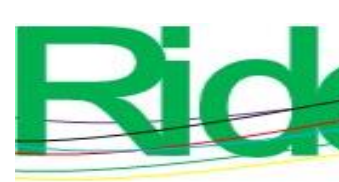

Revista Iberoamericana para la Investigación y el Desarrollo Educativo ISSN $2007-7467$

convierta en un factor protector que les ayude, como señala Damon (2009), a encontrar relevancia y significatividad en su experiencia académica.

Asimismo, los resultados del estudio permitieron diseñar la propuesta de una intervención educativa con enfoque de logoterapia para trabajar precisamente en la búsqueda del sentido de vida. Dicha intervención fue diseñada desde los principios educativos y para encontrar sentido. En su diseño se emplearon, además de las estrategias de enseñanzaaprendizaje, las técnicas de logoterapia. La estrategia podrá ser empleada para trabajar con todos los programas educativos de ciencias sociales, económico-administrativas y humanidades de una universidad pública en el sureste de México, siempre y cuando se consideren las características de cada grupo de estudiantes, sus estilos y canales de aprendizaje. Finalmente, los resultados mostraron que los materiales educativos a diseñarse y utilizarse durante la implementación de la propuesta no requerían de ser diseñados a partir de la edad y sexo.

\section{Conclusiones}

Este estudio representa una propuesta de trabajo innovadora ya que emplea el sentido de vida como herramienta para apoyar la formación integral del estudiantado. Mediante este tipo de educación centrada en el sentido se busca apoyar la búsqueda y el fortalecimiento del sentido de vida de los estudiantes de una universidad pública en el sureste de México. Se trata de una educación que, además de educar en el aspecto cognitivo, eduque desde la afectividad, las emociones, los aspectos sociales, físicos y valores-actitudinales, a fin de apoyar a los alumnos a autodescubrirse, reconocerse como personas únicas y valiosas, con la capacidad para tomar decisiones y responsabilizarse de estas, así como a autotrascender en la vida de los demás dejando huellas positivas, y también en el ejercicio de su profesión.

Asimismo, este estudio representa una alternativa que favorece la implementación del modelo educativo para la formación integral de la misma universidad, puesto que, a través del diseño e implementación de la intervención educativa para atender el fortalecimiento del sentido de vida, se están desarrollando estrategias para atender específicamente la dimensión valoral-actitudinal de los estudiantes desde los primeros años de licenciatura.

Cabe mencionar que se logró cumplir con el objetivo principal de este estudio, que fue diagnosticar el nivel de sentido de vida de los estudiantes de nuevo ingreso al nivel superior. Los resultados mostraron que la mayoría de los educandos presentaban niveles 


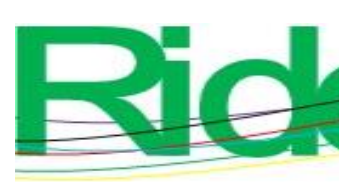

Revista Iberoamericana para la Investigación y el Desarrollo Educativo ISSN $2007-7467$

bajos de sentido de vida e incluso carecían de este factor por completo. Además, se encontró que las variables vinculadas con el programa educativo, edad y sexo no eran predictoras del sentido de vida. Dichos resultados también mostraron que no se requería de diseñar los materiales y recursos en función de la edad, sexo o licenciatura que se cursa. Y sobre todo, permitieron diseñar una intervención educativa como estrategia para atender este constructo, puesto que gracias al diagnóstico se pudo comprobar que

Los resultados reafirman la necesidad de que las instituciones educativas atiendan este constructo con la finalidad de favorecer la motivación de los alumnos, el desarrollo de sus talentos y enseñarlos a superar obstáculos para establecer metas y luchar por cumplirlas. No es otra cosa que proporcionarles herramientas de apoyo para adaptarse al ámbito universitario, a los requerimientos de este nivel educativo, así como para favorecer la trayectoria académica, promover el desarrollo de elevadas expectativas y que los estudiantes puedan convertirse en profesionales motivados e interesados por el bienestar de los demás desde la profesión que ejercen.

La escuela representa un contexto privilegiado cuyas figuras de autoridad deben enfocar sus esfuerzos en incorporar el sentido de vida como herramienta para contribuir en la formación integral de sus estudiantes, enseñarles a reconocerse como seres humanos valiosos, así como también a ser competentes en la disciplina que estudian para poder lograr sus sueños, metas e ideales, sobreponiéndose a las situaciones que inevitablemente tendrán que enfrentar debido a la naturaleza de cada área educativa, y poder gozar de una existencia personal y profesionalmente llena de sentido.

Está claro que la educación, además de enseñar conocimientos, debe enseñar a los educandos a convertirse en seres humanos reflexivos, críticos, creativos y capaces de comprender que el proceso de formación no tiene fin, sino que se despliega a lo largo de la vida, y que la importancia del sentido de vida radica en el hecho de que proporcionará satisfacción a sus vidas, relevancia en el desarrollo de las actividades que realizan, así como una fuerte motivación para desear continuar con su preparación académica, para superarse a sí mismos. Aquí se insta, pues, a que la escuela asuma el reto de incorporar programas de intervención educativa desde la logoterapia para trabajar en el fortalecimiento del sentido de vida de los estudiantes, de manera que estos deben constituirse como parte de la formación que deben recibir desde su primer ingreso al nivel superior, con la finalidad de potenciar sus capacidades y motivaciones para crecer en las diferentes esferas en las que se desenvuelven. 


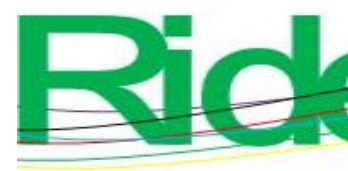
Revista Iberoamericana para la
Investigación y el Desarrollo Educativo
ISSN $2007-7467$

\section{Contribuciones a futuras líneas de investigación}

Como futuras líneas de investigación se propone llevar a cabo la implementación de intervenciones encaminadas en fortalecer el sentido de vida de los estudiantes de nuevo ingreso a la universidad y valorar el impacto de este en su formación a lo largo de su trayectoria académica, así como desarrollar estudios cualitativos enfocados en conocer cómo construyen su sentido de vida los educandos que ingresan a la universidad, los factores que influyen en la construcción de dicho sentido, así como la forma en la cual el sentido de vida influye en el establecimiento de metas académicas y profesionales de los educandos para apoyar la trayectoria académica.

\section{Referencias}

Andrade, D. (2018). Recursos noológicos y sentido de vida en estudiantes de psicología de una universidad privada de la ciudad de Lima. (Tesis de licenciatura). Universidad Inca Garcilaso de La Vega, Lima. Recuperado de http://repositorio.uigv.edu.pe/bitstream/handle/20.500.11818/3200/TESIS_David\%2 0Ricardo\%20Andrade\%20Mogrovejo.pdf?sequence=2\&isAllowed=y.

Armas, M. y López, A. (2018). El sentido de la vida: factor protector de ansiedad y depresión. Cauriensia. Revista Anual de Ciencias Eclesiásticas, 13, 57-73. Recuperado de http://www.cauriensia.es/index.php/cauriensia/article/view/XIII-EMO3/407.

Ascencio de García, L. (14 de septiembre de 2009). La logoterapia, más que una terapia: hacia una propuesta educativa. Logoforo. Recuperado de https://logoforo.com/lalogoterapia-mas-que-una-terapia-hacia-una-propuesta-educativa/.

Bernardez, J. (2018). Sentido de vida en estudiantes universitarios entre 18 y 20 años, estudiantes de primer ingreso de la carrera de administración de empresas de la Universidad Rafael Landivar, sede La Antigua Guatemala. (Tesis de licenciatura). Universidad Rafael Landívar, Antigua Guatemala. Recuperado de http://biblio3.url.edu.gt/publijrcifuente/TESIS/2018/05/42/Bernardez-Jeannefer.pdf.

Cabello, P. (2000). El sentido de la vida. Revista Semestral Pharos, Arte, Ciencia y Tecnología, 7(2), 95-99. Recuperado de https://www.redalyc.org/pdf/208/20807211.pdf. 


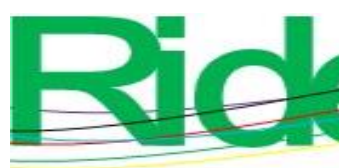

Revista Iberoamericana para la Investigación y el Desarrollo Educativo ISSN $2007-7467$

Cadavid, M. y Díaz, V. (2015). Sentido de vida de los universitarios. Revista Educación y Educadores, $\quad$ 18(3), 371-390. Recuperado de http://www.scielo.org.co/pdf/eded/v18n3/v18n3a01.pdf.

Castillo, J. (2018). Propósito de vida en estudiantes de un bachillerato público. (Tesis de licenciatura). Universidad Autónoma de Yucatán.

Crumbaugh, J. C. (1973). Everything to Again: A guide to Self-Fulfillment Through Logoanalysis. Berkeley, United States: Institute of Logotherapy Press.

Damon, W. (2009). The why question: Teachers can instill a sense of purpose. Education Next, 9(3), 84-94.

Fabry, J. (2001). Señales del camino hacia el sentido. México: Ediciones LAG.

Fabry, J. (2009). Señales del camino hacia el sentido. Descubriendo lo que realmente importa. México: Ediciones LAG.

Frankl V. (1997). Ante el vacío existencial. Barcelona, España: Herder.

Frankl, V. (2004). El hombre en busca del sentido. España: Herder

García, J., Gallego, J. y Pérez, E. (2009). Sentido de vida y desesperanza: un estudio empírico. Universitas Psychologica, 8(2), 447-454. Recuperado de www.scielo.org.co/pdf/rups/v8n2/v8n2a12.pdf

García, J., Martínez, E., Sellés, P. y Soucase, B. (2018). Sentido de vida y bienestar psicológico en adultos emergentes españoles. Revista Acta Colombiana de Psicología, 21(1), 206-2016. Recuperado de https://actacolombianapsicologia.ucatolica.edu.co/article/view/1292/1698.

Giraldo, P. (2014). El vacío existencial y la pérdida del sentido de vida en el sujeto posmoderno: retos para el cristianismo del siglo XXI. Cuestiones Teológicas, 41(96), 425-444. Recuperado de http://www.scielo.org.co/pdf/cteo/v41n96/v41n96a08.pdf.

Gómez del Campo, M., Medina, B. y Aguilar, D. (2011). Sentido de vida y éxito. Uaricha, Revista de Psicología, 8(17), 124-146. Recuperado de https://studylib.es/doc/7103535/sentido-de-vida-y-\%C3\%A9xito---revista-uaricha.

Lukas, E. (2006). Logoterapia. La búsqueda de sentido. México: Paidós

Magaña, L., Zavala, M., Ibarra, I., Gómez, M. y Gómez, M. (2004). El sentido de vida en estudiantes de primer semestre de la universidad de la Salle Bajío. Revista del Centro de Investigación. $6 \quad$ (22), 5-13 Recuperado de http://www.redalyc.org/articulo.oa?id=34202201 


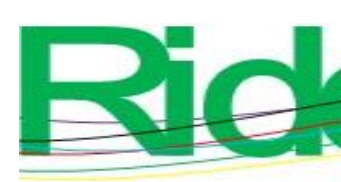

Revista Iberoamericana para la
Investigación y el Desarrollo Educativo
ISSN $2007-7467$

Maldonado, V., Arredondo, F. y De la Garza, J. (2010). El propósito de vida y la experiencia profesional en el alumno. Revista Investigación y Postgrado, 24(2), 317-340. Recuperado de www.redalyc.org/articulo.oa?id=65828402012.

Mallart, J., Mallart, A. y Valls, R. (2011). Educación para la responsabilidad en una pedagogía humanista. Ponencia presentada en el XII Congreso Internacional de Teoría de la Educación. Barcelona, marzo de 2011. Recuperado de https://www.researchgate.net/publication/323915326_Educacion_para_la_responsab ilidad_en_una_pedagogia_humanista/link/5ab262e8a6fdcc1bc0c0d576/download.

Martínez, E. (2007). Psicoterapia y sentido de vida. Colombia, Bogotá: Herder

Martínez, E. y Castellanos, C. (2013). Percepción del sentido de vida en universitarios colombianos. Revista Pensamiento Psicológico, 11(1), 71-82. Recuperado de http://revistas.javerianacali.edu.co/index.php/pensamientopsicologico/article/view/4 21.

Martínez, Y, Llantá, M. y Bayarre, H. (2018). Validación del test sentido de vida en pacientes adultos con cáncer. Revista Habanera de Ciencias Médicas, 17(5), 800-812. Recuperado de http://www.revhabanera.sld.cu/index.php/rhab/article/view/2407/2132.

Noblejas, A. (2000). Palabras para una vida con sentido. Bilbao, España: Desclée Brouwer.

Stramiello, C. (2006). Los sistemas educativos de América Latina y la recuperación del ser humano. En García, C. (coord.), Salven al hombre. Latinoamérica unida en la búsqueda de sentido (pp. 79-86). Buenos aires, Argentina: San Pablo.

Smedema, Y. y Franco, M. (2018). Resiliencia, ansiedad y sentido de la vida en estudiantes universitarios. Revista Cauriensia, 13, 87-106. Recuperado de http://www.cauriensia.es/index.php/cauriensia/article/view/XIII-EMO5/408.

Tinto, V. (1975). Definir la deserción: una cuestión de perspectiva. Revista de la Educación Superior, Publicaciones de la ANUIES (71).

Wojtyla, K. (2005). El hombre y su destino. Madrid, España: Palabra.

Xirau, R. (1987). Introducción a la historia de la filosofía. Ciudad de México, México: Universidad Nacional Autónoma de México. 